\title{
Monolayer Graphene-covered Grids Enable 2.6-Å Single-particle Cryo-EM Reconstruction of 52-kDa Streptavidin
}

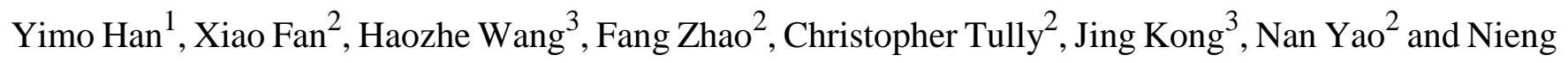
$\mathrm{Yan}^{2}$

${ }^{1}$ Rice University, Houston, Texas, United States, ${ }^{2}$ Princeton University, Princeton, New Jersey, United States, ${ }^{3}$ Massachusetts Institute of Technology, Boston, Massachusetts, United States

Single-particle cryogenic electron microscopy (cryo-EM) represents the cutting-edge technology for the structural determination of biomacromolecules. However, technical challenges associated with cryo-EM sample preparation limit its ability from achieving higher resolution for a broader range of targets, especially for small proteins. In order to improve the sample quality, new designs of the cryo-EM grids, aimed at preserving thin, uniform vitrified ice and improving protein adsorption, have been considered a promising approach to achieving a higher resolution with the minimal amount of materials and data.

Here, we demonstrate a 2.6- $\AA$ single-particle reconstruction of 52-kDa streptavidin (Fig. 1) [1-3]. This is so far the highest resolution structure of the smallest protein by cryo-EM. The structure was achieved by using a high-yield, monolayer graphene-supporting cryo-EM grid, which allows for effective protein adsorption and thin vitrified ice [1]. We fabricated graphene cryo-EM grids by transferring continuous monolayer graphene from its original substrate, a copper foil, to a Quantifoil holey carbon Au grid using an organic molecule-assisted transfer method (Fig. 2a). We employed UV/ozone, which uses ultraviolet (UV) irradiation to generate a small amount of ozone gas to gently oxidize sample surfaces (Fig. $2 \mathbf{b}$ ). UV/ozone has the advantage of adding functional groups to graphitic surfaces at a slow, and thus, controllable rate, therefore, fine-tuning the surface properties of graphene (Fig. 2c). Using this approach, we have achieved uniform and clean monolayer graphene with a high yield of suspended areas ( $99 \%$ on average) (Fig. 2d). In addition to streptavidin, graphene grids also effectively increase the density of other examined soluble, membrane, and lipoproteins (Fig. 2e-i), affording the opportunity for structural investigation of challenging proteins which cannot be produced in large quantity.

In summary, we developed a robust approach to produce graphene-covered grids for cryo-EM and achieved a 2.6- $\AA$ structure of 52-kDa streptavidin. Our technique paves the way for higher quality and more general cryo-EM sample preparation for near-atomic resolution cryo-EM. In addition, our method employs only simple tools that most structural biology laboratories can access. We expect our method to benefit the cryo-EM community by improving the sample preparation process [5].
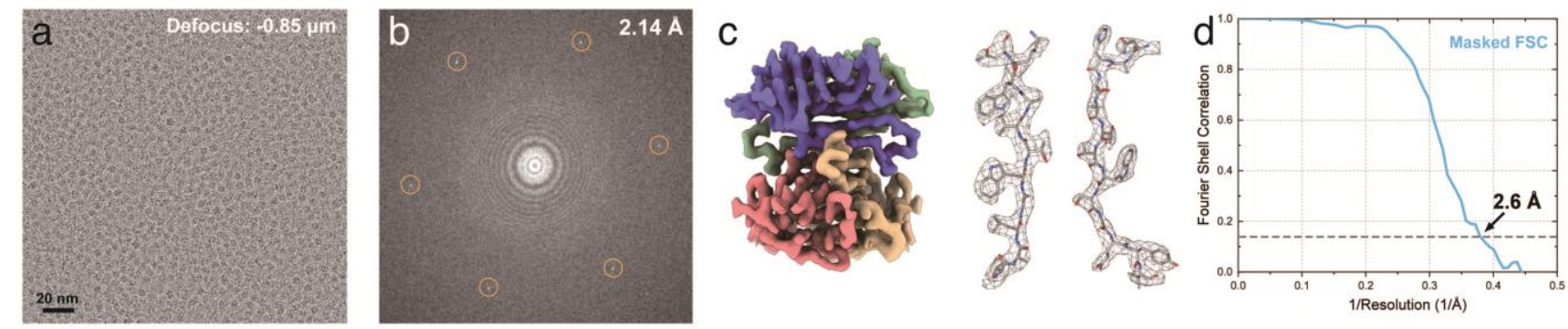

Figure 1. Single-particle cryo-EM reconstruction of 52-kDa streptavidin at a $2.6-\AA$ resolution. a, Cryo-EM micrograph of 52-kDa streptavidin particles with a small defocus value $(-0.85 \mu \mathrm{m})$. b, Fourier 
transform of a with graphene reciprocal lattice circled in orange. c, Single-particle reconstruction of streptavidin at 2.6- $\AA$ r resolution [2,3]. d, Gold standard FSC (criteria 0.143) curve of the masked map.

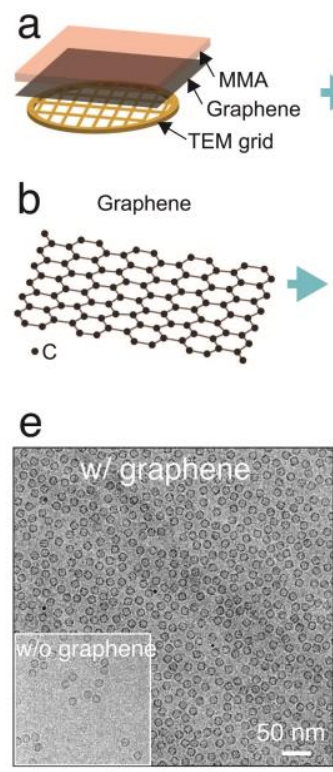

Apoferritin
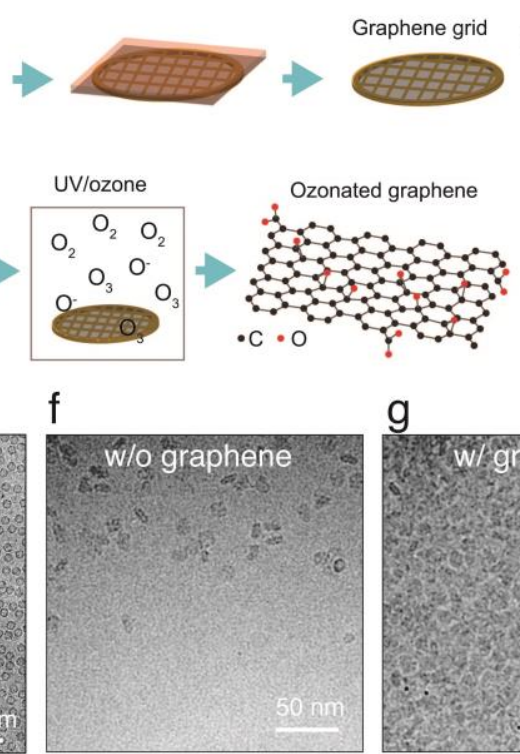

$\mathrm{NaChBac}$

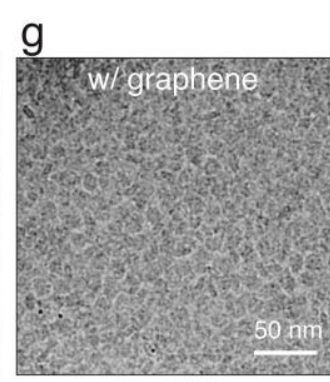

$\mathrm{NaChBac}$

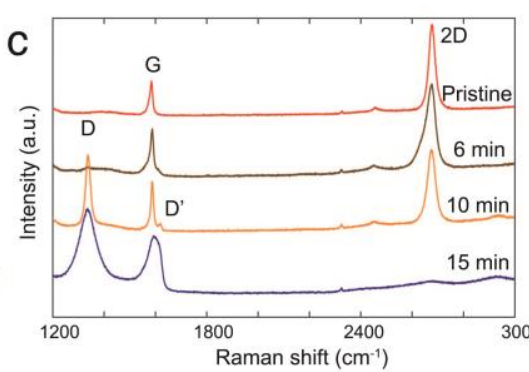

$\mathrm{h}$

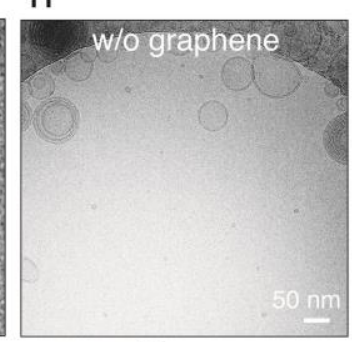

Liposome
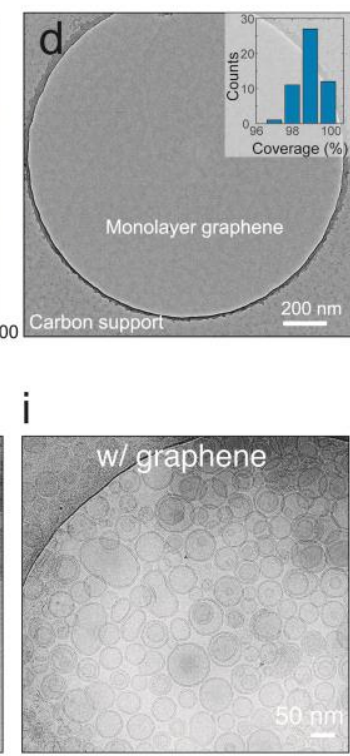

Liposome

Figure 2. Fabrication and applications of high-yield monolayer graphene grids for cryo-EM. a, Schematic summary of the grid fabrication process (more details in ref. 1). b, Schematic of graphene surface treatment by UV/ozone, which can convert graphene to hydrophilic oxygenated graphene derivatives. c, Raman spectroscopy shows that 10-15 min UV/ozone treatment can effectively functionalize graphene surface. d, TEM image of suspended graphene over a hole in the Quantifoil Au grid. The inset displays the statistics of graphene yield ( $99 \%$ on average). e, Cryo-EM micrograph of apoferritin on graphene grids, compared to the same sample on holey carbon grids (inset). f-g, Cryo-EM micrographs of a bacterial sodium channel $(\mathrm{NaChBac})$ on holey carbon grids (f) and graphene grids $(\mathbf{g})$. $\mathbf{h}-\mathbf{i}$, Cryo-EM micrographs of liposomes on holey carbon grids (h) and graphene grids (i).

\section{References}

[1] Y. Han et al. PNAS, 117 (2020), p. 1099-1014.

[2] Y. Han, X. Fan, and N. Yan, EMD-20907 (2019).

[3] Y. Han, X. Fan, and N. Yan, EMPIAR-10335 (2019).

[4] Funded by the Dean for Research Innovation Fund for New Ideas in the Natural Sciences from Princeton University.

[5] Thank the Princeton Institute for the Science and Technology of Materials (PRISM). 